The phenomenon in question has, of course, no connection with precession or nutation, but is concerned only with a variation of the position of the axis of rotation of the earth within the earth itself. Its effect is to periodically increase the latitude of places on one side of the earth and simultaneously diminish that of those on the opposite side of the earth by amounts of the order of 30 or 40 feet. In other words, the pole is 30 or 40 feet nearer Washington, and the same farther from Peking at one time than another.

Mr. Chandler's first announcement of his discovery in 1891 began as follows:

"In the determination of the latitude of Cambridge, Mass., with the Almucantar about six years and a half ago, it was shown that the observed values, arranged according to nights of observation, exhibited a decided and curious progression throughout the series, the earlier values being small, the latter ones large, and the range from November, 1884, to April, 1885, being about four-tenths of a second. There is no known or imaginable instrumental or personal cause for this phenomenon, yet the only alternative seemed to be an inference that the latitude had actually changed. This seemed at the time too bold an inference to place upon record, and I therefore left the results to speak for themselves."

He gives further data of his own on the subject ending to support the hypothesis of a change of latitude, and then refers to European observations contemporaneous with his own as follows:

"Curiously enough Dr. Küstner, in his determination of the latitude from a series of observations coincident in time with those of the Almucantar, came upon similar anomalies, and his results, published in 1888, furnish a counterpart to those I had pointed out in 885. The verification afforded by the recent paralle determinations at Berlin, Prague, Potsdam, and Pulkowa, which show a most surprising and satisfactory accordance as to the character of the change in range and periodicity with the Almucantar results, has led me to make further investigations on the subject."

Mr. Chandler then proceeded to give detailed re sults of the investigations, which he summed up as follows:

"The general result of a preliminary discussion is to show a revolution of the earth's pole in a period of 427 days, from west to east, with a radius of 30 feet, measured at the earth's surface. Assuming provisionally, for the purpose of statement, that this is a mo tion of the north pole of the principal axis of inertia about that of the axis of rotation, the direction of the former from the latter lay toward the Greenwich meridian abcut the beginning of the year 1890. This, with the period of 427 days, will serve to fix approximately the relative positions of these axes at any other time for any given meridian. It is not possible ai this stage of the investigation to be more prēcise, s there are facts which appear to show that the rotation is not a perfectly uniform one, but is subject to secular change, and perhaps irregularities, within brief spaces of time."

These results of observation fell squarely across the long-accepted theory of Euler, according to which the variation of latitude, if any, must be a uniform one of ten months, and also contradicted the result of careful reduction of excellent observations from which it had been concluded that no sensible wandering of the pole did in fact take place. The theoretical difficulty was, indeed, soon partly removed by Prof. Newcomb, who pointed out that the fluidity of the ocean and the elasticity of the earth had been neglected in deriving ten months as the theoretical period of a possible rotation of the earth's pole, but at the same time he professed himself unable to account on the principles of dynamics for a variation of the period of the inequality, unattended by an alteration of its amplitude during the preceding half century, conclusions which had come from recomputations which Mr. Chandler had in the meantime made of the classic observations of Bradley in 1728, and others of different periods of the nineteenth century.

Under the spur of the theoretical difficulties stated by Prof. Newcomb, Mr. Chandler collected an immense mass of evidence on the subject, involving the reduction of more than 33,000 observations made by nine different methods, comprising the work of seventeen observatories, distributed over both the northern and southern hemispheres, and covering the interval of time from 1728 to 1890 . From this great array of evidence the fact of the wandering of the pole was not only clearly confirmed, but also the variation of its period and amplitude came out without question, and an insight was gained as to the causes of this baffling phenomenon as follows:

"The observed variation of the latitude is the resultant curve arising from two periodic fluctuations superposed upon each other. The first of these, and in general the more considerable, has a period of about 427 days, and a semiamplitude of about 0.12 second. The second has an annual period with a range variable between 0.04 and 0.20 second during the last half century. During the middle portion of this in- terval, roughly characterized as between 1860 and 1880 , the value represented by the lower limit has prevailed, but before and after these dates, the higher

"As the resultant of these two motions, the effective variation of latitude is subject to a systematic alternation in a cycle of seven years' duration, resulting from the commensurability of the two terms. According as they conspire or interfere, the total range varies between two-thirds of a second, as a maximum, to but a few hundredths of a second, generally speaking, as a minimum.

"In consequence of the variability of the coefficient of the annual term above mentioned, the apparent average period between 1840 and 1855 approximated to 380 or 390 days; widely fluctuated from 1855 to 1865; from 1865 to 1885 was very nearly 427 days with minor fluctuations; afterward increased to near 440 days."

Mr. Chandler expressed these results mathematically by an equation in which the variation of latitude is given as the sum of two periodic terms, and he found that this expression very closely represented all the observations he had so laboriously collected.

The valuable consequences of this discovery of $\mathrm{Mr}$. Chandler's are numerous. First of all, they showed why certain series of observations made with the most painstaking care at the national observatories of the United States, Great Britain, and Russia had exhibited discordancies which had led to distrust of the very best pieces of apparatus, and had involved laborious, costly, and fruitless efforts to remove what now were found to be non-existing defects. In some instances even the reputation for accuracy of able astronomers had been clouded by such inexplicable discordances in their work, which, in the light of the new discovery, now prove only the evidences of the faithfulness and accuracy of these observers. Again, as to the constant of aberration, on which depends one important method of determining the distance of the

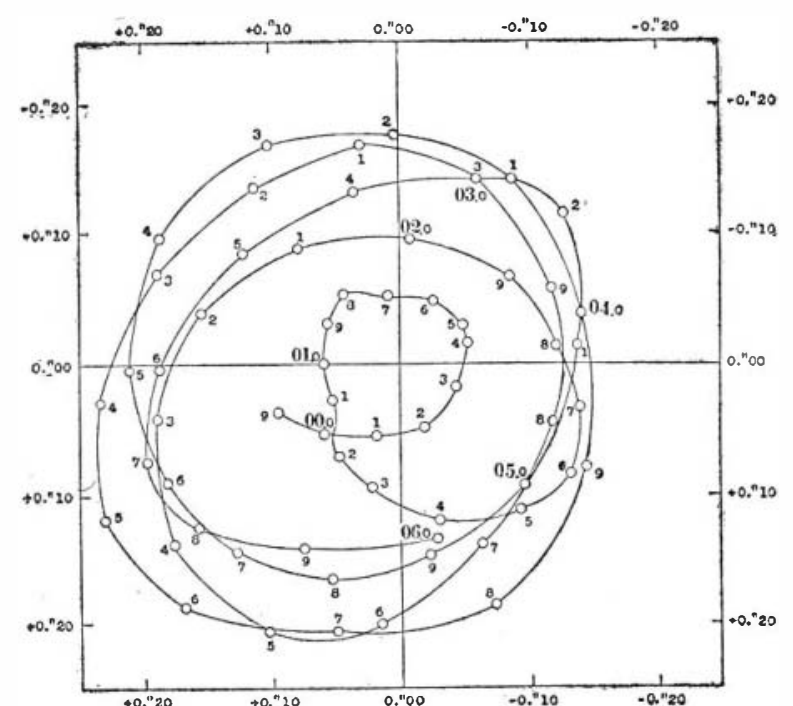

MOVEMENT OF EARTH'S AXIS, 1899 TO 1906

sun (that great astronomical quantity on which all the conclusions as to the distances, masses, and other elements of the solar system hang, together with all the exact predictions depending on solar theory), it proves that the determinations of this important conthe variations of latitude. Prof. Turner, in his Astronomical Discovery, has the following passage indicating still another possible ffect of the wandering of the pole:

"If the axis of the earth is executing small oscillations of this kind, there should be an effect upon the tides; the liquid ocean should feel the wobble of the earth's axis in some way; and an examination of the tidal registers showed that there was, in fact, a distinct effect. It may cause some amusement when I say that the rise and fall are only a few inches in any case, but they are unmistakable evidences that the earth is not spinning smoothly, but has this kind of unbalanced vibration, which I have compared to the vibrations felt by passengers on an imperfectly engineered twin-screw steamer. A more sensational effect is that apparently earthquakes are more numerous at the time when the vibration is greatest. We remark that the vibration waxes and wanes much as that of the steamer waxes and wanes if the twin screws are not running quite together. Now, the passenger on the steamer would be prepared to find that breakages would be more numerous during the times of vigorous oscillation, and it seems probable that in a similar way the little cracks of the earth's skin, which we call great earthquakes, are more numerous when these unbalanced vibrations are at their maximum; that is to say, about once every seven years. This result is scarcely yet worthy our complete confidence, for our observations of earthquakes have only very recently been reduced to proper order; but if it should turn out to be true, it is scarcely necessary to add any words of mine to demonstrate the importance of this rather unexpected result of latitude variation."
The existence of the two principal factors in the variation of latitude, substantially as discovered by Mr. Chandler, àre fully confirmed by the observations of the international latitude stations which have now been continued for six years. The period of the principal one is at present thought to be 437 days, however. The accompanying figure shows in a graphical form exactly how the earth's axis has been wobbling within the earth in these last six years. It is taken from a recent paper on the subject by Prof. Th. Albrecht, who, with Dr. B. Wanach, has reduced and published the international observations. In the figure the scale of motion is indicated in seconds of arc, which can be translated into feet or meters by remembering that one second of arc at the pole is about 100 feet, or 30 meters, so that the diameter of the larger curves is about 40 feet, or 12 meters. The time is indicated in years and tenths of a year by the figures adjoining the curves themselves. Thus, beginning with 1899.9 the curve passes over the years 1900,1901 , etc., and ends at 1906.0. It is plainly shown by this irregular curve what an apparently lawless wobble of the earth's axis is produced by the joint action of two periodic factors, one of about 437 days and one annual, the first of constant and the second of variable amplitude. Since 1890 it has been found that five of the long periods are almost exactly equal to six of the shorter, so that the whole movement nearly repeats itself every six years. It is interesting, though not conclusive, to note that the severe earthquakes of fime of maxis soon after the tim of the pole, which accords with the hypoth
upon by Prof. Turner, as already cited.

\section{THE USES OF CALCIUM IN ALLOYS.*}

\author{
By Arthur E. Pratr.
}

Calcium is a silvery white metal readily oxidized in moist air. It is very light (sp. gr. 1.52), fairly malleable, has a high specific heat, and is a good conductor of electricity. It is about as hard as aluminium, but at 400 deg. C. becomes as soft as lead. It is volatile, and can be sublimed in vacuo between 700 deg. C. and 800 deg. C., and melts at the latter temperature. It is a very powerful reducing agent.

The chief effects of alloying calcium with other metals are to produce brittleness, crystallization, and hardness; to promote oxidation and disintegration on exposure to air; to confer the power of decomposing
water and in other ways increasing the chemical activity.

The author's experiments confirm Roberts-Austen's observation that the presence of small amounts of metals of high atomic volume will cause deterioration of the physical properties of metals of low atomic volume. The atomic volume of calcium is high (25.4), and the effect of small amounts on other metals is decidedly prejudicial, provided that the metals in question are pure. The experiments were conducted in a converse manner to Roberts-Austen's, i. e., the constant was a metal of high atomic volume (calcium) instead of being low (gold). In the course of the work the following observations were made: Wher an alloy is made of calcium and some metal which possesses a chemical property in common with it, an increased activity in the manifestation of that property is noticed in the alloy. This increase appears to be greater than would be obtained by the simple admixture of a more active metal, the presence of calcium usually increasing the activity of the other metal. In some cases the alloy is more active than either of its constituents.

Further, the chemical properties of calcium appear to be more pronounced in an alloy with a metal having an atomic value closely approaching that of calcium than they are in an alloy of the same percentage with a metal having a much lower atomic value. The two metals in question should be about equally active when unalloyed in the particular property with respect to which they are to be compared. It is probable that both these principles are general, and not confined to calcium, although more extanded research on these lines would be desirable.

The most promising applications of calcium are as a reducing agent and for the refining of metals. In the latter case it acts in three distinct ways: (1) By reducing oxides and sulphides; (2) by eliminating dissolved gases; (3) by forming compounds with certain impurities, thus rendering them less deleterious. All three modes of action are strikingly shown in the case of copper. A suitable addition of calcium will remedy "dry" or "sulphury" copper, give a sound casting, and give a soft and tough ingot with prohibitive proportions of bismuth or antimony, besides restoring ordinary over-poled copper to tough pitch. If excess of calcium is present, however, it induces brittleness on its own account. With one or two doubtful exceptions, no alloy of calcium has shown any promise of commercial utility so far as physical properties are concerned, its only likely application in this direction being its hardening property. 